\title{
Gating Classical Information Flow via Equilibrium Quantum Phase Transitions
}

\author{
Leonardo Banchi, ${ }^{1}$ Joaquín Fernández-Rossier, ${ }^{2,3}$ Cyrus F. Hirjibehedin, ${ }^{1,4,5}$ and Sougato Bose ${ }^{1}$ \\ ${ }^{1}$ Department of Physics and Astronomy, University College London (UCL), London WC1E 6BT, United Kingdom \\ ${ }^{2}$ Quantalab, International Iberian Nanotechnology Laboratory, 4715-330 Braga, Portugal \\ ${ }^{3}$ Departamento de Fsica Aplicada, Universidad de Alicante, 03690 San Vicente del Raspeig, Spain \\ ${ }^{4}$ London Centre for Nanotechnology, UCL, London WC1H OAH, United Kingdom \\ ${ }^{5}$ Department of Chemistry, UCL, London WC1H OAJ, United Kingdom \\ (Received 1 July 2016; published 5 April 2017; corrected 7 April 2017)
}

\begin{abstract}
The development of communication channels at the ultimate size limit of atomic scale physical dimensions will make the use of quantum entities an imperative. In this regime, quantum fluctuations naturally become prominent and are generally considered to be detrimental. Here, we show that for spin-based information processing, these fluctuations can be uniquely exploited to gate the flow of classical binary information across a magnetic chain in thermal equilibrium. Moreover, this information flow can be controlled with a modest external magnetic field that drives the system through different many-body quantum phases in which the orientation of the final spin does or does not reflect the orientation of the initial input. Our results are general for a wide class of anisotropic spin chains that act as magnetic cellular automata and suggest that quantum phase transitions play a unique role in driving classical information flow at the atomic scale.
\end{abstract}

DOI: 10.1103/PhysRevLett.118.147203

As the size of information processing platforms decreases, quantum mechanics becomes more prominent, both as a resource [1] and also as an intrinsic source of fluctuations $[2,3]$. Given the dramatic recent advances in the ability to engineer finite structures of interacting atomic or molecular spins [4-15], it becomes important to explore whether finite nanoscale chains of interacting quantum entities in thermal equilibrium are a viable on-chip connector for transmitting bits using either their charge or spin degrees of freedom. However, despite the potential of these structures for lowdissipation spin-based information technology $[9,11,15]$, there has been no investigation of the capacity of their robust thermal (static) states to convey classical information, in particular, in the sense of Shannon's quantitative theory [16]. Moreover, in the atomic regime, quantum fluctuations, rather than being merely noise, play a fundamental role and can actively drive a phase transition in a many-body system $[17,18]$. Here, we show that these quantum phase transitions can produce striking changes in the information transfer capacity and thereby demonstrate a fully quantum methodology for gating the classical information flow through a large class of magnetic chains.

We consider a generic setup for transmitting classical digital information through the equilibrium state of a quantum spin chain (Fig. 1). As in recent experiments [9], the magnetic island on the left has uniaxial anisotropy so that it only has two ground states and therefore encodes one bit of classical information. This input is inserted into a spin chain via exchange coupling to the first quantum spin $S_{1}$. Every other spin $S_{j}$ of the chain is coupled to its nearest neighbors. The magnetic island is sufficiently large as to be described with a classical magnetization $S_{0}$ and to make the back action of the quantum spins negligible. The logical state of the island can be controlled independently, for example, by using external magnetic pulses [9]. In this system, the output is defined by the orientation of the last spin $S_{L}$, where $L$ counts the number of quantum spins in the chain. The read-out can be realized using spin-polarized tunneling [19]. Typically, the initialization and measurement times are much slower than the equilibration time, so during the output measurement, the chain is in its equilibrium state.

If we ignore both quantum and thermal fluctuations, so that the spin chain is described with classical Ising spins at $T=0$, with two equivalent ground states, perfect transmission occurs from the island to the opposite boundary: fixing the logical state of the first spin $S_{1}$ selects one of the two ground states for the entire chain. It can be readily seen that thermal fluctuations, at the classical level, destroy this ideal picture. To quantify this, we make use of Shannon's seminal work [16], where he showed that the maximum rate at which information can be transmitted over a memoryless communications channel with arbitrarily small decoding error is given by the so-called channel capacity $C$ (Supplemental Material [20]). The capacity is 1 for errorfree channels, 0 for fully broken ones, and in some simple cases is a function of the probability $P_{f}$ of bit-flip errors at the output. Applying this formalism to the classical Ising model at inverse temperature $\beta=1 /\left(k_{B} T\right)$ (Supplemental Material [20]), where each spin can point either up or down, we find $P_{f}=0$ at $T=0$, and the chain perfectly transfers information. However, at high temperature, the capacity goes down as $C \simeq$ $\exp \left(-4 L e^{-2 \beta J}\right)$, where $J$ is the strength of the Ising coupling. Therefore, thermal fluctuations limit the maximum length of the chain for reliable information transfer to $L \approx e^{2 \beta J}$.

Whereas it is possible in principle to suppress classical fluctuations by reducing $T$, the same will not be true for 
quantum spin fluctuations, making a study of their effects imperative. Once we switch from classical to quantum systems, the reconstruction of the output density matrix is necessary to determine the ultimate rate of classical information transfer [21-25] (see also the Supplemental Material [20]). However, motivated by an experimental perspective in adatom chains, we focus on a simpler scheme, where the digital output is encoded into the sign of the magnetization $\left\langle S_{L}^{z}\right\rangle$ of the last spin, as described above, and we study the information capacity when the channel spins are described with the anisotropic Heisenberg Hamiltonian

$$
\begin{gathered}
\mathcal{H}_{\text {chain }}=J \sum_{n=1}^{L-1} \vec{S}_{n} \cdot \vec{S}_{n+1}+\sum_{n=1}^{L} \mathcal{H}_{n}, \\
\mathcal{H}_{n}=D\left(S_{n}^{z}\right)^{2}+E\left[\left(S_{n}^{x}\right)^{2}-\left(S_{n}^{y}\right)^{2}\right]+\vec{B} \cdot \vec{S}_{n},
\end{gathered}
$$

where (Fig. 1) $L$ is the length of the chain, $S_{n}^{\alpha}$ is the quantum spin operator along the direction $\alpha=x, y, z$ acting on the $n$th spin, $\vec{B}$ is the magnetic field (with the Bohr magneton and the Landé $g$ factor absorbed into the definition of $\vec{B}$ ), $D$ is the (axial) zero field splitting, $E$ is the planar (transverse) anisotropy of the crystal field interaction, and $J$ is the exchange integral between neighboring sites. This Hamiltonian can describe a variety of different physical phenomena, depending on the relative value of the exchange interaction and anisotropy, as well as the value of the spin $S$. Importantly, these quantities can be tuned experimentally, and the model has been used to successfully describe experimentally realized spin chains $[6,8,11,12]$.

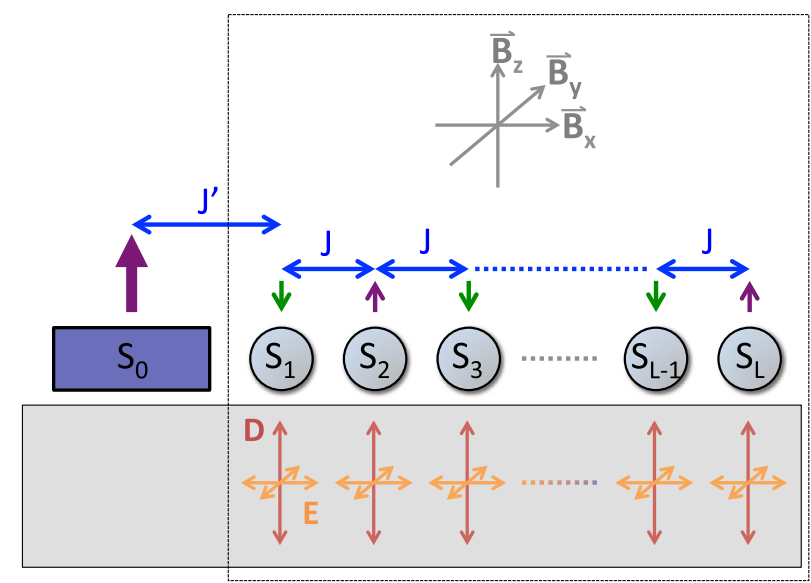

FIG. 1. A spin chain and its interactions on a surface. Schematic illustrating individual atomic spins $S_{1} \ldots S_{L}$ (gray balls) in a chain on a surface (solid gray rectangle) coupled by an interaction with strength $J$ (blue arrows). The first spin $S_{1}$ is coupled to a large, semiclassical spin $S_{0}$ (purple rectangle) via an interaction $J^{\prime}$ (blue arrow). Also shown are the axial and transverse anisotropy terms, $D$ (red arrows) and $E$ (orange arrows), respectively, and the components of an externally applied magnetic field $\vec{B}$ (gray arrows). Green and purple arrows indicate the magnetic orientation of the spins for an antiferromagnetic coupling.
The effect of a large magnetic island, whose orientation can be tuned externally [9], can be described by a classical magnetic field $B_{0}=J^{\prime} S_{0}$ pointing along the $z$ direction, so that the total Hamiltonian is $\mathcal{H}=\mathcal{H}_{\text {chain }}+B_{0} S_{1}^{z}$. Here, we will focus on antiferromagnetic (AFM) chains (i.e., $J>0$ ), which have shown considerable stability at low temperatures [11]. Although we find that ferromagnetic systems generally have a higher capacity than their AFM counterparts, this capacity is highly sensitive to external fields in the $z$ direction (Supplemental Material [20]).

We first consider a chain with isotropic spin-spin couplings, with $\vec{B}=E=D=0$. Figure 2 shows the digital channel capacity (Supplemental Material [20]) of such finite length quantum spin chains, where we use a density matrix renormalization group (DMRG) algorithm [26] to compute the ground state. It is apparent that the channel capacity is smaller for chains of lower spins, with isotropic $S=1 / 2$ systems being particularly poorly suited for information transfer. Even for larger spins, the capacity clearly decreases as the length of the chain increases (Fig. 2) because the perturbation due to the local coupling with the island, which breaks the rotational symmetry of the state of the spin chain, is not able to propagate along the chain.

To stabilize only the two states $\left|m_{n}^{z}= \pm S\right\rangle$, it is therefore natural to consider spin systems with $S \geq 1$ and large negative $D$. As seen in Fig. 2, as we increase the uniaxial anisotropy, the channel capacity increases and becomes less

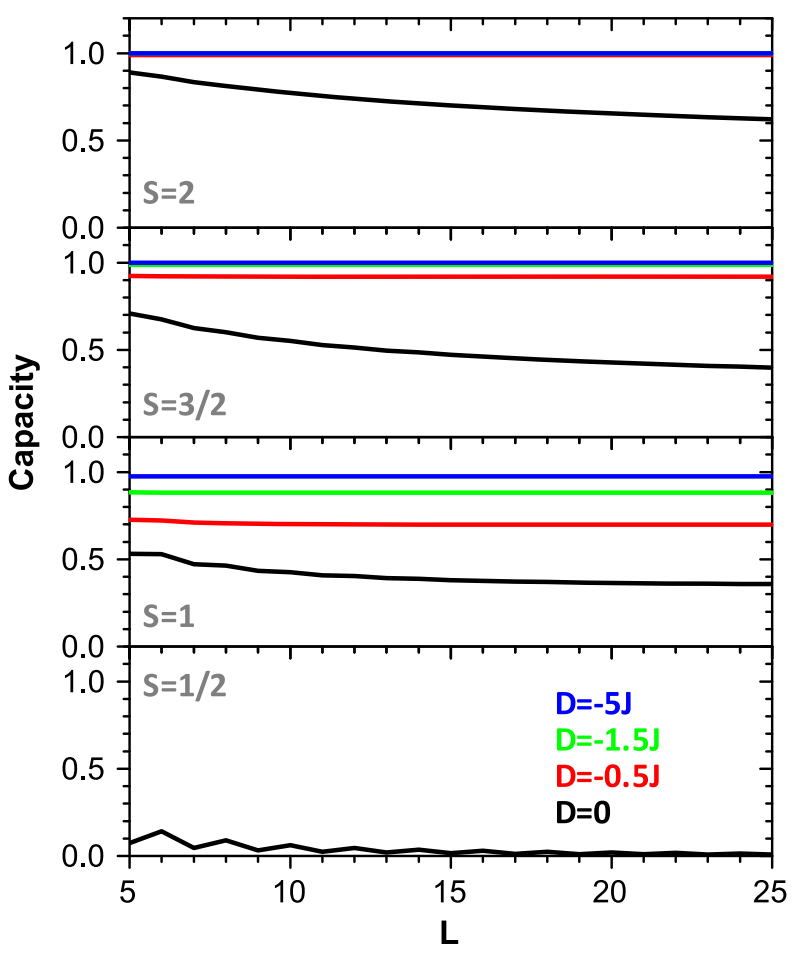

FIG. 2. Impact of axial anisotropy on channel capacity. Capacity vs chain length for Heisenberg-coupled spin chains with $E=0$ and $B_{0}=100 J$ for $S=2, S=3 / 2, S=1$, and $S=1 / 2$, as calculated using DMRG for $D=0$ (black), $D=$ $-0.5 \mathrm{~J}$ (red), $D=-1.5 \mathrm{~J}$, and $D=-5 \mathrm{~J}$ (blue). 
dependent on the channel length. For sufficiently large uniaxial anisotropy $(D=-5 J, E=0)$, the channel is similar to classical Ising spins at $T=0$. Therefore, both temperature and antiferromagnetic flip-flop interactions driven by exchange can compromise the channel capacity.

We now show that there is yet another factor that affects the channel capacity decisively: quantum spin tunneling of the individual spins, driven in this system by the transverse anisotropy [27] $E$. As shown in Fig. 3, where we keep $E / D$ fixed and vary $D / J$, the capacity remains relatively large (above 0.9) for $S=3 / 2$ and $S=2$, even with finite values of $E$ once a sufficiently large $(|D| \gtrsim J)$ axial anisotropy is present. In sharp contrast, however, the case of $S=1$ (Fig. 3) exhibits a rapid decrease in capacity down to zero above a critical value. The role played by the in-plane anisotropy $E$, very different from the effect of the uniaxial anisotropy $D$, can be understood analytically, in the limit $|D / J| \gg 1$ and $D<0$. In this case, each spin $S \geq 1$ is approximated [28]

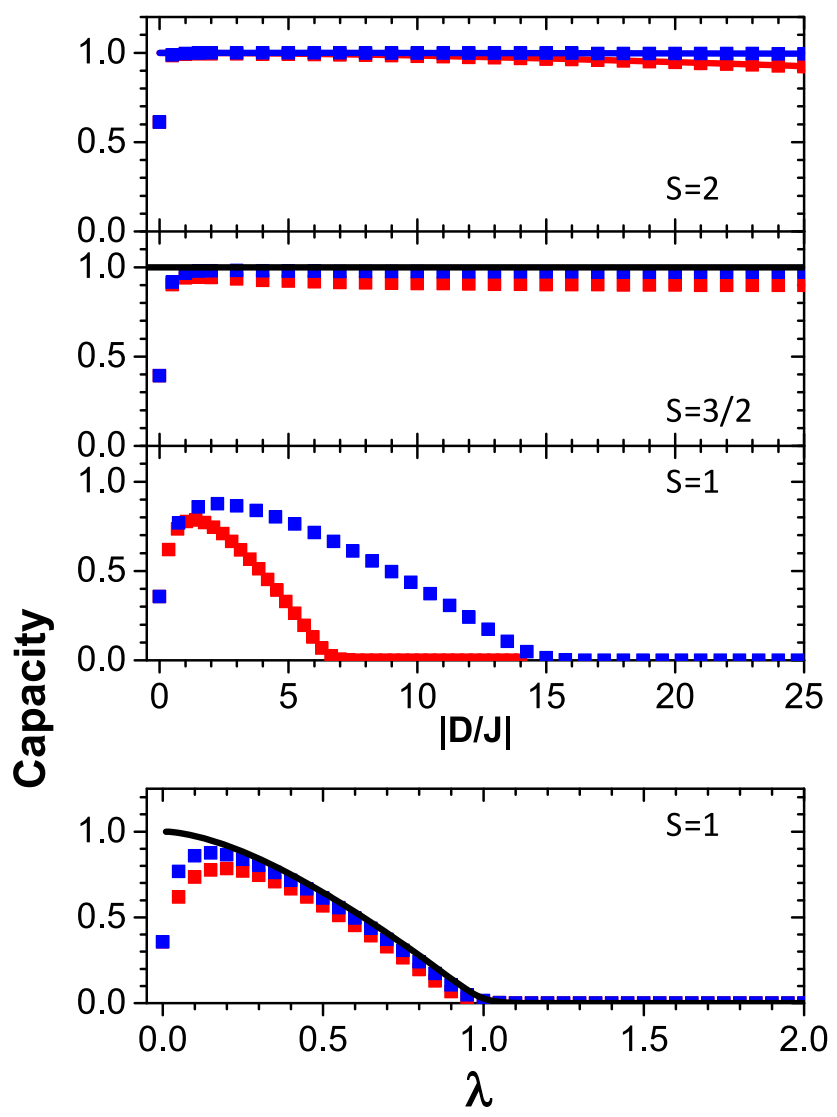

FIG. 3. Impact of quantum fluctuations on spin chain capacities. (upper panel) Capacity of a spin chain with $L=25$, $B_{0}=J$, and $\vec{B}=0$, as obtained from DMRG calculations for $S=2, S=3 / 2$ and $S=1$, plotted as a function of $|D / J|$ for $D=-7 E$ (red squares) and $D=-15 E$ (blue squares). The ratio $\eta=D / E$ is kept fixed as $|D / J|$ increases. (lower panel) The above results for $S=1$ are plotted against $\lambda$. Solid lines, in both panels, show the predictions of the effective model (Table I) with the same parameters. A single black line is used when the results are independent of $\eta$. with an effective two-level system $| \pm S\rangle$, namely with a pseudospin $\tau=1 / 2$. At the single spin level, Kramers theorem ensures that these two levels are degenerate for half-integers spins, but are, in general, split due to single-spin quantum spin tunneling (SS-QST) in the case of integer spins. This splitting acts as an effective field in the pseudospin space, so that the resulting model for the channel is the quantum Ising model (QIM) in a transverse field $\lambda$ (see Table I), which is one of the paradigmatic systems for the study of quantum phase transitions [17]. The QIM has two distinct phases. The interaction dominated phase, with $|\lambda|<1$, has two ordered ground states, characterized in the thermodynamic limit $(L \rightarrow \infty)$ by long-range correlations and a nonzero order parameter $\left\langle\tau_{n}^{z}\right\rangle$. For $|\lambda|>1$, the field dominated phase, there is a unique paramagnetic ground state that results in a quantum disordered phase with shortrange correlations and a vanishing order parameter.

Within the QIM, we can find an analytical expression (Supplemental Material [20]) for the magnetization of the output spin after the local perturbation introduced by the island

$m_{L}^{z} \equiv\left\langle\tau_{L}^{z}\right\rangle \simeq \begin{cases}\operatorname{sgn}(\mu)(-1)^{L+1} \sqrt{1-\lambda^{2}} & \text { if }|\lambda|<1, \\ 0 & \text { if }|\lambda|>1,\end{cases}$

when $L \gg 1$, where $\mu \propto B_{0} / J$ (see Table I). Equation (3) shows that in the thermodynamic limit $m_{L}^{z}$ is a nonanalytic function of $\mu$ and depends only on its sign, while for finite systems this nonanalytic behavior is smoothened. Finite-size effects are negligible far from the critical point and can be estimated from conformal invariance at $\lambda \approx 1[30,31]$. From Eq. (3), the capacity $C$ can be evaluated, and we find in the thermodynamic limit that $C=$ $1+\sum_{ \pm}\left(1 \pm \sqrt{1-\lambda^{2}} / 2\right) \log _{2}\left(1 \pm \sqrt{1-\lambda^{2}} / 2\right)$ for $|\lambda|<1$, while the capacity is zero when $m_{L}^{z}=0$. This shows that the chain acts as a "wire" able to carry digital information from the island to the distant opposite end only in the ordered phase, while when $|\lambda|>1$, the appearance of a unique gapped ground state blocks the information flow.

TABLE I. Low-energy effective Hamiltonian for antiferromagnetic chains in the subspace $\left|m_{n}^{z}= \pm S\right\rangle$ when $D<0, \vec{B} \| \hat{x}$ and $\vec{B}_{0} \| \hat{z}$. The operators $\tau_{n}^{\alpha}$ are effective spin- $1 / 2$ Pauli operators acting on the low-energy subspace. The effective theory has been obtained with the theory presented in Ref. [29] using a second order expansion in $\epsilon=(D / J)^{-1}$ (third order for $S=2$ ), assuming that $(E / J) \simeq \mathcal{O}\left(\epsilon^{\eta}\right),\left(B_{x} / J\right) \simeq \mathcal{O}\left(\epsilon^{\beta}\right)$, where $0<\eta<1,0<$ $\beta<1$ and keeping only the dominant terms. Within these assumptions, no $\epsilon^{2}$ corrections are present for $S=1$.

\begin{tabular}{llc}
\hline \hline & \multicolumn{2}{c}{$\mathcal{H}_{\mathrm{eff}}=\mu \tau_{1}^{z}+\sum_{n}\left(\lambda \tau_{n}^{x}+\tau_{n}^{z} \tau_{n+1}^{z}\right)$} \\
\hline Spin 1 & $\mu \simeq B_{0} / J$ & $\lambda \simeq E / J-B_{x}^{2} / 2|D| J$ \\
Spin $\frac{3}{2}$ & $\mu \simeq 2 B_{0} / 3 J$ & $\lambda \simeq-2 B_{x} E / 3|D| J+B_{x}^{3} / 12 D^{2} J$ \\
& & $+B_{x} E^{2} / 3 D^{2} J$ \\
Spin 2 & $\mu \simeq B_{0} / 2 J$ & $\lambda \simeq-3 E^{2} / 8|D| J+5 B_{x}^{2} E / 24 D^{2} J$ \\
& & $-B_{x}^{4} / 96|D|^{3} J$ \\
\hline \hline
\end{tabular}


The dependence of $\lambda$ on the physical parameters of the chain is very different for $S=1$ (Table I) and can be used to account for the very different behavior shown in Fig. 3 . In particular, for $S=1$ as $\lambda \simeq E / J$ is increased, the system undergoes a quantum phase transition. In contrast, for $S=3 / 2$, there is no zero field splitting, in agreement with Kramers theorem, and $\lambda=0$ for $\vec{B}=0$. For $S=2$, $\lambda \simeq E^{2} / D J$, which is much smaller than for $S=1$. As a result, for fixed $E / D$, the fluctuation dominated paramagnetic phase can only be achieved when $D / J$ is very large, $D / J>(E / D)^{-2}$ - see also Supplemental Material Sec. II. B and Fig. S1 [20]. As seen in Fig. 3, the effective model presented in Table I is in excellent agreement with the DMRG results (for $|D| \gtrsim J$ ), showing that $S=1$ systems are unique in providing a flexible system where the flow of information depends on quantum phases.

Having unveiled the relevant role played by quantum fluctuations and SS-QST permits us to devise a strategy to control the spin chain channel capacity using externally tunable parameters. For this, we use the fact that, at the single-spin level, the application of a magnetic field $B_{x}$ along the hard axis modulates the quantum spin tunneling splitting [32,33], which acts as an effective magnetic field $\lambda$ in the pseudospin space, and thereby also affects the collective state in the spin chain. This is seen in Fig. 4, where we consider a spin chain with $S=1$ at two different values of the anisotropy: $E=0.5 \mathrm{~J}$ in Fig. 4(a), and $E=$ $1.5 \mathrm{~J}$ in Figs. 4(b) and 4(c). Without applied fields, the chain of Fig. 4(a) is in the ordered phase $(\lambda=0.5)$ with an imperfect capacity and that of Fig. 4(b) is in the quantum disordered phase $(\lambda=1.5)$ with vanishing channel capacity. In this nontrivial regime, as seen in Fig. 4(c), the AFM configuration is preserved only around the magnetic island while far from this boundary all the spin components $m_{n}^{\alpha}$ are zero, meaning that each spin is highly entangled with the others [34]. Moreover, at $\lambda=1$ the magnetization profile along the chain can also be obtained from conformal invariance [30,31]. As we start increasing $B_{x}$, the SS-QST decreases in both cases, and the channel capacity is improved. This effect is even more dramatic in Fig. 4(b), where, in an initially disordered chain, $\lambda\left(B_{x}\right)$ is decreased below the critical value $\lambda=1$ and magnetic order along the $z$ axis is established due to application of a magnetic field along the orthogonal $x$ axis. At the optimal field $B_{c}$, it is $\lambda=0$, and the channel capacity is maximal [see also Fig. 4(c)]. As the magnetic field $B_{x}$ is further increased, the system eventually reaches the trivial limit where the Zeeman energy dominates all other energy scales, and the capacity to transmit information vanishes. Our numerical calculations show that the dramatic effect of the transverse magnetic field on the channel capacity is remarkable for anisotropic $S=1$ chains, the only ones for which $\lambda$ is independent of the uniaxial anisotropy $D$ at $B_{x}=0$. The channel capacity of chains with $S=3 / 2$ spins shows a weak dependence on $B_{x}$, expected from the functional dependence of $\lambda\left(B_{x}\right)$ (see Table I), while in higher-spin

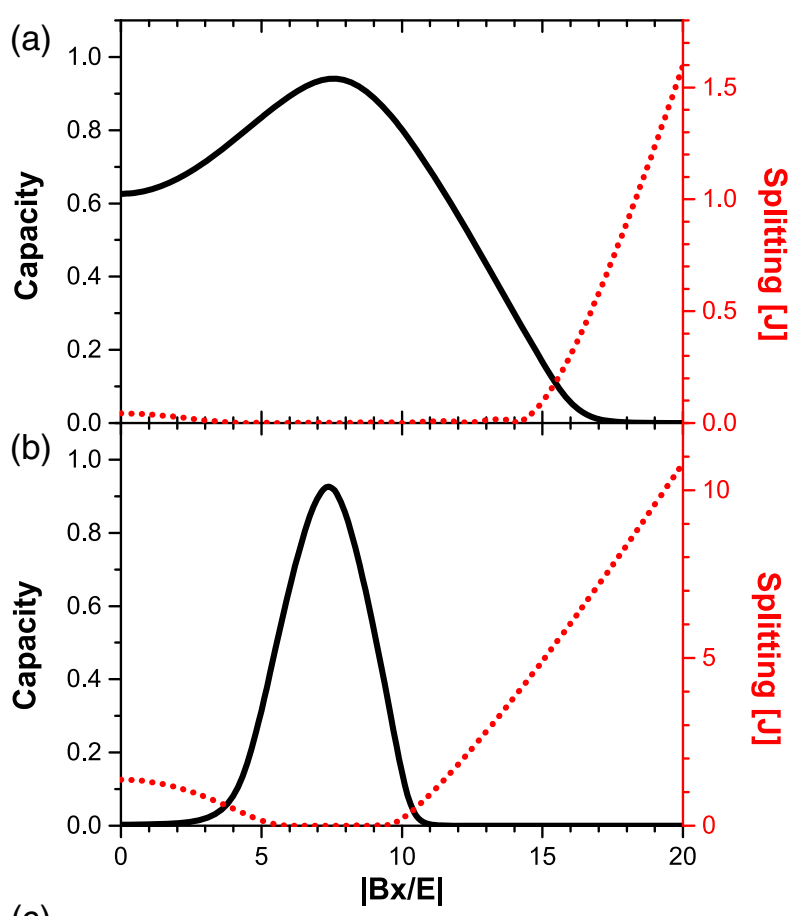

(c)



FIG. 4. Gating capacity via quantum fluctuations with an externally applied magnetic field. (a) Capacity (solid black line) and splitting between the two lowest energy states (dotted red line) for an $S=1$ spin chain with $L=6, D=-25 E$, and $E=0.5 \mathrm{~J}$, as obtained from exact diagonalization, plotted as a function of $\left|B_{x} / E\right|$. The calculation for the capacity uses $B_{0}=J$, while the calculation for the gap uses $B_{0}=0$. (b) Same as panel (a) with $E=1.5 \mathrm{~J}$, so that quantum fluctuations completely destroy the capacity at $B_{x}=0$. (c) Spin configurations in the $x z$ plane for a chain with the same parameters as for panel (b). The initial, larger spin represents the magnetic island.

systems, only the regime $\lambda \simeq 0$ is accessible due to the damping of the QST splitting for large $|D|$. To the leading orders in perturbation theory (see Supplemental Material [20] for more general arguments), maximal capacity $(\lambda=0)$ corresponds to the "diabolic points" [33], where the QST splitting of each spin vanishes. On the other hand, in a chain, the energy splitting is small (vanishes in the thermodynamic limit) in the whole interval $|\lambda|<1$ [see Figs. 4(a) and 4(b)], so the regions of nonzero capacity correspond to quantum ordered chains with (almost) degenerate ground states.

The theoretical framework introduced here uses the standard metric from information theory for the first time to analyze the transfer of classical information along a quantum magnetic chain in thermal equilibrium. Our approach highlights the deep relationship between quantum 
magnetic phases and information transmission. Thus, we find a way to take advantage of the competition between various energy scales-such as uniaxial and in-plane anisotropy as well as exchange interactions and Zeeman coupling - to devise a nontrivial method to tune magnetic order, and thereby the channel capacity in the spin chain, using external magnetic fields. Given the typical values of anisotropy and spin coupling that have been observed for magnetic atoms on surfaces [4,6,9,12,35-38] $(|D| \sim 0.1-1 \mathrm{meV},|E| \leq|D| / 3$, and $|J| \sim 0.1-10 \mathrm{meV})$, $B_{c}$ can be below $10 \mathrm{~T}$ and therefore easily accessible with current experimental probes. Future work will consider the additional effects of electronic coupling of the spins in the chain to the substrate, which can be tuned by fabricating the structures on top of superconducting [39] or even insulating materials, the latter of which would require the use of force-based microscopy for the fabrication and readout. Our results also suggest that more complex quasi-onedimensional spin structures, such as spin chains with next nearest neighbor coupling or spin ladders, may also exhibit intriguing phenomena that can be used to manipulate their classical information capacity. Finally, we note that STMbased pump-probe techniques [40] may be extended in the future with multiple tips to enable experimental exploration of the propagation of the dynamics along the chain.

Numerical data that support the findings of this paper are available by following the link in Ref. [41].

We thank Ian Affleck, Cristian Batista, Adrian Feiguin, Andrew Fisher, Chris Lutz, and Sebastian Loth for stimulating discussions, some of which were during SPICE workshop "Magnetic Adatoms as Building Blocks for Quantum Magnetism" and the MPI-IBM workshop in Almaden. L. B. and S. B. have received funding for this research from the European Research Council under the European Union's Seventh Framework Programme (FP7/2007-2013)/ERC Grant agreement No. 308253 PACOMANEDIA; C.F.H. from the Leverhulme Trust (Grant No. RPG-2012-754) and EPSRC (Grant No. EP/ M009564/1); J. F. R. from the UCLQ Visitors Program.

[1] M. A. Nielsen and I. L. Chuang, Quantum Computation and Quantum Information (Cambridge University Press, Cambridge, England, 2010).

[2] M. H. Devoret, Quantum fluctuations in electrical circuits, in Quantum Fluctuations: Les Houches Session LXIII, 1995, edited by S. Reynaud, E. Giacobino, and J. Zinn-Justin (Elsevier, Amsterdam, The Netherlands, 1997), p. 351.

[3] H.-S. Wong, IBM J. Res. Dev. 46, 133 (2002).

[4] P. Gambardella, A. Dallmeyer, K. Maiti, M. Malagoli, W. Eberhardt, K. Kern, and C. Carbone, Nature (London) 416, 301 (2002).

[5] H. J. Lee, W. Ho, and M. Persson, Phys. Rev. Lett. 92, 186802 (2004).

[6] C. F. Hirjibehedin, C. P. Lutz, and A. J. Heinrich, Science 312, 1021 (2006).
[7] D. Kitchen, A. Richardella, J.-M. Tang, M. E. Flatté, and A. Yazdani, Nature (London) 442, 436 (2006).

[8] X. Chen, Y.-S. Fu, S.-H. Ji, T. Zhang, P. Cheng, X.-C. Ma, X.-L. Zou, W.-H. Duan, J.-F. Jia, and Q.-K. Xue, Phys. Rev. Lett. 101, 197208 (2008).

[9] A. A. Khajetoorians, J. Wiebe, B. Chilian, and R. Wiesendanger, Science 332, 1062 (2011).

[10] A. A. Khajetoorians, J. Wiebe, B. Chilian, S. Lounis, S. Blügel, and R. Wiesendanger, Nat. Phys. 8, 497 (2012).

[11] S. Loth, S. Baumann, C. P. Lutz, D. Eigler, and A. J. Heinrich, Science 335, 196 (2012).

[12] A. Spinelli, B. Bryant, F. Delgado, J. Fernández-Rossier, and A. F. Otte, Nat. Mater. 13, 782 (2014).

[13] S. Nadj-Perge, I. K. Drozdov, J. Li, H. Chen, S. Jeon, J. Seo, A. H. MacDonald, B. A. Bernevig, and A. Yazdani, Science 346, 602 (2014).

[14] B. Weber, S. Mahapatra, H. Ryu, S. Lee, A. Fuhrer, T. Reusch, D. Thompson, W. Lee, G. Klimeck, L. C. Hollenberg et al., Science 335, 64 (2012).

[15] M. Menzel, Y. Mokrousov, R. Wieser, J. E. Bickel, E. Vedmedenko, S. Blügel, S. Heinze, K. von Bergmann, A. Kubetzka, and R. Wiesendanger, Phys. Rev. Lett. 108, 197204 (2012).

[16] C. Shannon, Bell Syst. Tech. J. 27, 379 (1948).

[17] A. Dutta, G. Aeppli, B. K. Chakrabarti, U. Divakaran, T. F. Rosenbaum, and D. Sen, Quantum Phase Transitions in Transverse Field Models (Cambridge University Press, Cambridge, England, 2015).

[18] S. Sachdev, Quantum Phase Transitions (Wiley Online Library, 2007).

[19] R. Wiesendanger, Rev. Mod. Phys. 81, 1495 (2009).

[20] See Supplemental Material at http://link.aps.org/ supplemental/10.1103/PhysRevLett.118.147203 for an extended definition of the capacity of classical and quantum channels, the analytic solution of the effective model, and its extension to ferromagnetic systems. The Supplementary Material contains also extended discussions on the relationship between capacity and ground state degeneracy, the appearance of an effective field for $S=1$, the validity of the effective model and the evaluation of the capacity of classical Ising chains.

[21] A. S. Holevo, Prob. Peredachi Inf. 9, 3 (1973) [Prob. Inf. Trans. 9, 177 (1973)].

[22] V. Giovannetti and R. Fazio, Phys. Rev. A 71, 032314 (2005).

[23] V. Giovannetti, R. García-Patrón, N. Cerf, and A. Holevo, Nat. Photonics 8, 796 (2014).

[24] V. Giovannetti, S. Lloyd, L. Maccone, and J. H. Shapiro, Nat. Photonics 7, 834 (2013).

[25] K. Banaszek, Nat. Photonics 6, 351 (2012).

[26] U. Schollwöck, Ann. Phys. (Amsterdam) 326, 96 (2011).

[27] F. Delgado and J. Fernández-Rossier, Phys. Rev. Lett. 108, 196602 (2012).

[28] F. Delgado, S. Loth, M. Zielinski, and J. Fernndez-Rossier, Europhys. Lett. 109, 57001 (2015).

[29] N. Jia, L. Banchi, A. Bayat, G. Dong, and S. Bose, Sci. Rep. 5, 13665 (2015).

[30] F. Iglói and H. Rieger, Phys. Rev. Lett. 78, 2473 (1997).

[31] T. W. Burkhardt and T. Xue, Phys. Rev. Lett. 66, 895 (1991).

[32] A. Garg, Europhys. Lett. 22, 205 (1993).

[33] W. Wernsdorfer and R. Sessoli, Science 284, 133 (1999). 
[34] L. Amico, R. Fazio, A. Osterloh, and V. Vedral, Rev. Mod. Phys. 80, 517 (2008).

[35] P. Gambardella, S. Rusponi, M. Veronese, S. Dhesi, C. Grazioli, A. Dallmeyer, I. Cabria, R. Zeller, P. Dederichs, K. Kern, C. Carbone, and H. Brune, Science 300, 1130 (2003).

[36] C. F. Hirjibehedin, C.-Y.Lin, A. F. Otte, M. Ternes, C. P. Lutz, B. A. Jones, and A. J. Heinrich, Science 317, 1199 (2007).

[37] A. A. Khajetoorians, T. Schlenk, B. Schweflinghaus, M. dos Santos Dias, M. Steinbrecher, M. Bouhassoune, S. Lounis,
J. Wiebe, and R. Wiesendanger, Phys. Rev. Lett. 111, 157204 (2013).

[38] B. Bryant, A. Spinelli, J. J. T. Wagenaar, M. Gerrits, and A. F. Otte, Phys. Rev. Lett. 111, 127203 (2013).

[39] B. Heinrich, L. Braun, J. Pascual, and K. Franke, Nat. Phys. 9, 765 (2013).

[40] S. Loth, M. Etzkorn, C. P. Lutz, D. Eigler, and A. J. Heinrich, Science 329, 1628 (2010).

[41] See https://doi.org/10.6084/m9.figshare.c.3278864. 